\title{
Lie symmetry analysis and conservation laws for the time fractional simplified modified Kawahara equation
}

https://doi.org/10.1515/phys-2018-0042

Received August 26, 2017; accepted February 10, 2018

\begin{abstract}
In this work, Lie symmetry analysis for the time fractional simplified modified Kawahara (SMK) equation with Riemann-Liouville (RL) derivative, is analyzed. We transform the time fractional SMK equation to nonlinear ordinary differential equation (ODE) of fractional order using its Lie point symmetries with a new dependent variable. In the reduced equation, the derivative is in the Erdelyi-Kober (EK) sense. We solve the reduced fractional ODE using a power series technique. Using Ibragimov's nonlocal conservation method to time fractional partial differential equations, we compute conservation laws (Cls) for the time fractional SMK equation. Some figures of the obtained explicit solution are presented.
\end{abstract}

Keywords: time fractional SMK, Lie symmetry, exact solutions, conservation laws

PACS: $02.20 . \mathrm{Sv} ; 02.20 . \mathrm{Hj} ; 02.20 . \mathrm{Qs} ; 02.60 . \mathrm{Cb}$

\section{Introduction}

Symmetry analysis has many applications in the field of science and engineering. Lie's method is one of the global and efficient methods for investigating analytical solutions and symmetry properties of nonlinear partial differential equations (NLPDEs) [1-17]. Fractional calculus has

\footnotetext{
*Corresponding Author: Dumitru Baleanu: Cankaya University, Department of Mathematics, Öğretmenler Cad. 1406530, Ankara/Türkiye. Institute of Space Sciences, Magurele, Bucharest, Romania, E-mail: dumitru@cankaya.edu.tr

Mustafa Inc: Firat University, Science Faculty, Department of Mathematics, 23119,, Elaziǧ/Turkey, E-mail: minc@firat.edu.tr Abdullahi Yusuf: Firat University, Science Faculty, Department of Mathematics, 23119,, Elaziǧ/Turkey,

E-mail: yusufabdullahi@fud.edu.ng

Aliyu Isa Aliyu: Firat University, Science Faculty, Department of Mathematics, 23119,, Elaziǧ/Turkey, E-mail: aliyu.isa@fud.edu.ng
}

been successfully used to explain many complex nonlinear phenomena and dynamic processes in physics, engineering, electromagnetics, viscoelasticity, and electrochemistry [18-34].

Generally, physical phenomenon might depend on its current state and on its historical states, which can be modelled successfully by applying the theory of derivatives and integrals of fractional order $[35,36]$. Due to this, several analytical techniques are used to derive exact, explicit, and numerical solutions of nonlinear fractional partial differential equations (FPDEs) [30-34]. We find very few studies of symmetry analysis for FPDEs and their group properties are not plainly understood [37-41].

In other words, Cls are universally known to possess an important role in the analysis of NLPDEs from a physical viewpoint [42] . If the considered system has Cls, then its integrability will be possible $[43,44]$. Noether theorem supplies us with a strategic idea for constructing $\mathrm{Cls}$ of NLPDEs so long as the Noether symmetry associated with the Lagrangian is known for Euler-Lagrange equations [45]. Nevertheless, there are some techniques in the literature for obtaining the Cls of the NLPDEs, that do not have the Lagrangian [46-47].

Time fractional NLPDEs come from classical NLPDEs by replacing its time derivative with a fractional derivative. In the present work, we study Lie symmetry analysis, explicit solution using the power series technique and Ibragimov's nonlocal Cls [48] for the time fractional SMK equation given by

$$
\frac{\partial^{\alpha} u}{\partial t^{\alpha}}+\beta u^{2} u_{x}+\gamma u_{x x x x x}=0,
$$

in Eq. (1), $0<\alpha \leq 1$, and $\beta$ and $\gamma$ are arbitrary constants, and $\alpha$ is the order of the fractional time derivative. If $\alpha=1$, Eq. (1) reduces to the classical SMK equation which was considered for exact travelling wave solutions and $\mathrm{Cls}$ in [49-51]. Moreover, one can find more details on the construction of analytical, exact, numerical solutions, and other information for classical NLPDEs, in [52-69]. 


\section{Preliminaries}

Consider the RL fractional derivative $[70,71]$ given by

$$
D^{\alpha} f(t)=\left\{\begin{array}{lr}
\frac{d^{n} f}{d t^{n}}, & \alpha=n, \\
\frac{d}{d t^{n}} I^{n-\alpha} f(t), & 0 \leq n-1<\alpha<n,
\end{array}\right.
$$

where $n$ is a natural number and $I^{\mu} f(t)$ is the RL fractional integral of order $\mu$ given by

$$
\begin{aligned}
& I^{\mu} f(t)=\frac{1}{\Gamma(\mu)} \int_{0}^{t}(t-s)^{\mu-1} f(s) d s, \mu>0 \\
& I^{\mu} f(t)=f(t)
\end{aligned}
$$

and $\Gamma(z)$ represents Gamma function.

Consider time-fractional PDEs as below

$$
\partial_{t}^{\alpha} u=F\left(t, x, u, u_{x}, u_{x x}, u_{x x x}, \ldots\right), \quad(0<\alpha<1) .
$$

Given a one-parameter Lie group of infinitesimal transformations of the form

$$
\begin{aligned}
\bar{t} & =t+\epsilon \xi^{2}(t, x, u)+O\left(\epsilon^{2}\right), \\
\bar{x} & =x+\epsilon \xi^{1}(t, x, u)+O\left(\epsilon^{2}\right), \\
\bar{u} & =u+\epsilon \eta(t, x, u)+O\left(\epsilon^{2}\right), \\
\frac{\partial^{\alpha} \bar{u}}{\partial \bar{t}} & =\frac{\partial^{\alpha} u}{\partial t^{\alpha}}+\epsilon \eta_{\alpha}^{0}(t, x, u)+O\left(\epsilon^{2}\right), \\
\frac{\partial \bar{u}}{\partial \bar{x}} & =\frac{\partial u}{\partial x}+\epsilon \eta^{x}(t, x, u)+O\left(\epsilon^{2}\right), \\
\frac{\partial^{2} \bar{u}}{\partial \bar{x}^{2}} & =\frac{\partial^{2} u}{\partial x^{2}}+\epsilon \eta^{x x}(t, x, u)+O\left(\epsilon^{2}\right), \\
\frac{\partial^{3} \bar{u}}{\partial \bar{x}^{3}} & =\frac{\partial^{3} u}{\partial x^{3}}+\epsilon \eta^{x x x}(t, x, u)+O\left(\epsilon^{2}\right), \\
\frac{\partial^{4} \bar{u}}{\partial \bar{x}^{4}} & =\frac{\partial^{4} u}{\partial x^{4}}+\epsilon \eta^{x x x x}(t, x, u)+O\left(\epsilon^{2}\right), \\
\frac{\partial^{5} \bar{u}}{\partial \bar{x}^{5}} & =\frac{\partial^{5} u}{\partial x^{5}}+\epsilon \eta^{x x x x x}(t, x, u)+O\left(\epsilon^{2}\right),
\end{aligned}
$$

where

$$
\begin{aligned}
\eta^{x} & =D_{x}(\eta)-u_{x} D_{x}\left(\xi^{1}\right)-u_{t} D_{t}\left(\xi^{2}\right), \\
\eta^{x x} & =D_{x}\left(\eta^{x}\right)-u_{x t} D_{x}\left(\xi^{1}\right)-u_{x x} D_{t}\left(\xi^{2}\right), \\
\eta^{x x x} & =D_{x}\left(\eta^{x x}\right)-u_{x x t} D_{x}\left(\xi^{1}\right)-u_{x x x} D_{t}\left(\xi^{2}\right), \\
\eta^{x x x x} & =D_{x}\left(\eta^{x x x}\right)-u_{x x x t} D_{x}\left(\xi^{1}\right)-u_{x x x x} D_{t}\left(\xi^{2}\right), \\
\eta^{x x x x x} & =D_{x}\left(\eta^{x x x x}\right)-u_{x x x x t} D_{x}\left(\xi^{1}\right)-u_{x x x x} D_{t}\left(\xi^{2}\right),
\end{aligned}
$$

In Eq. (5), $D_{X}$ is the total differential operator defined by

$$
D_{x}=\frac{\partial}{\partial x}+u_{x} \frac{\partial}{\partial u}+u_{x x} \frac{\partial}{\partial u_{x}}+\ldots .
$$

The corresponding Lie algebra of symmetries consists of a set of vector fields of the form

$$
X=\xi^{1} \frac{\partial}{\partial x}+\xi^{2} \frac{\partial}{\partial t}+\eta \frac{\partial}{\partial u} .
$$

The vector field Eq. (6) is a Lie point symmetry of Eq. (3) provided

$$
\left.P^{\alpha, 5} r X(\nabla)\right|_{\nabla=0}=0 .
$$

Also, the invariance condition yields [72] gives

$$
\left.\xi^{2}(t, x, u)\right|_{t=0}=0
$$

and the $\alpha^{\text {th }}$ extended infinitesimal related to RL fractional time derivative with Eq. (8) is given by $[54,55]$.

$$
\begin{aligned}
\eta_{\alpha}^{0}=\frac{\partial^{\alpha} \eta}{\partial t^{\alpha}}+\left(\eta_{u}-\alpha D_{t}\left(\xi^{2}\right)\right) \frac{\partial^{\alpha} u}{\partial t^{\alpha}}-u \frac{\partial^{\alpha} \eta_{u}}{\partial t^{\alpha}}+\mu \\
-\sum_{n=1}^{\infty}\left(\begin{array}{l}
\alpha \\
n
\end{array}\right) D_{t}^{n}\left(\xi^{1}\right) D_{t}^{\alpha-n}\left(u_{x}\right) \\
+\sum_{n=1}^{\infty}\left[\left(\begin{array}{l}
\alpha \\
n
\end{array}\right) \frac{\partial^{\alpha} \eta_{u}}{\partial t^{\alpha}}-\left(\begin{array}{c}
\alpha \\
n+1
\end{array}\right) D_{t}^{n+1}\left(\xi^{2}\right)\right] D_{t}^{\alpha-n}(u),
\end{aligned}
$$

in Eq. (9)

$$
\begin{aligned}
\mu=\sum_{n=2}^{\infty} & \sum_{m=2}^{n} \sum_{k=2}^{m} \sum_{r=0}^{k-1}\left(\begin{array}{l}
\alpha \\
n
\end{array}\right)\left(\begin{array}{l}
n \\
m
\end{array}\right)\left(\begin{array}{l}
k \\
r
\end{array}\right) \frac{1}{k !} \frac{t^{n-\alpha}}{\Gamma(n+1-\alpha)} \\
& \times[-u]^{r} \frac{\partial^{m}}{\partial t^{m}}\left[u^{k-r}\right] \frac{\partial^{n-m+k}}{\partial t^{n-m} \partial u^{k}} .
\end{aligned}
$$

It is worth noting that, $\mu=0$ if the infinitesimal $\eta$ is linear in $u$, due to the presence of $\frac{\partial \eta^{k}}{\partial u^{k}}$, where $k \geq 2$ in Eq. (10).

Definition 2.1. The function $u=\Theta(x, t)$ is an invariant solution of Eq. (3) corresponding to the infinitesimal generator Eq. (6) provided that

1. $u=\Theta(x, t)$ satisfies Eq. (3).

2. $u=\Theta(x, t)$ is an invariant surface of Eq. (5), that is to say

$$
\xi^{2}(x, t, \Theta) \Theta_{t}+\xi^{1}(x, t, \Theta) \Theta_{x}=\eta(x, t, \Theta) .
$$

\section{Lie symmetries and reduction for Eq. (1)}

Suppose that Eq. (1) is an invariant under Eq. (5), we have that

$$
\bar{u}_{\bar{t}}^{\alpha}+\beta \bar{u}^{2} \bar{u}_{\bar{x}}+\gamma \bar{u}_{\bar{x} \bar{x} \bar{x} \bar{x} \bar{x}}=0 \text {, }
$$


so that, $u=u(x, t)$ satisfies Eq. (1). Using Eq. (5) in Eq. (11), we get the invariant equation

$$
\eta_{\alpha}^{0}+\left(2 \beta u u_{x}\right) \eta+\left(\beta u^{2}\right) \eta^{x}+\gamma \eta^{x x x x x}=0 .
$$

Putting the values of $\eta_{\alpha}^{0}, \eta^{x}$ and $\eta^{x x x x x}$ from Eq. (5) and Eq. (9) into Eq. (12) and isolating coefficients in partial derivatives with respect to $x$ and power of $u$, we get

$$
\begin{gathered}
\partial_{t}^{\alpha} \eta-u \partial_{t}^{\alpha} \eta_{u}-\beta u^{2} \eta_{x}-\gamma \eta_{x x x x x}=0, \\
\left(\begin{array}{c}
\alpha \\
n
\end{array}\right) \partial_{t}^{n}(\eta)-\left(\begin{array}{c}
\alpha \\
n+1
\end{array}\right) D_{t}^{n+1}\left(\xi^{2}\right)=0, n=1,2, \ldots \\
\xi_{u}^{1}=\xi_{u}^{2}=\xi_{t}^{1}=\xi_{x}^{2}=\eta_{u u}=0, \\
5 \xi_{x}^{1}-\alpha \xi_{t}^{2}=0 .
\end{gathered}
$$

Solving these equations, we get:

$$
\xi^{1}=c_{1}+x \alpha c_{2}, \quad \xi^{2}=5 t c_{2}, \quad \eta=-2 \alpha u c_{2},
$$

where $c_{1}$ and $c_{2}$ are arbitrary constants. Thus infinitesimal symmetry group for Eq. (1) is spanned by the two vector fields

$$
X_{1}=\frac{\partial}{\partial x}, \quad X_{2}=x \alpha \frac{\partial}{\partial x}+5 t \frac{\partial}{\partial t}-2 u \alpha \frac{\partial}{\partial u} .
$$

The similarity variables for the infinitesimal generator $X_{2}$ can be obtained by solving the following equations

$$
\frac{d x}{\alpha x}=\frac{d t}{5 t}=-\frac{d u}{2 \alpha u} .
$$

Solving the above equations, we get

$$
z_{1}=x t^{-\frac{\alpha}{5}}, z_{2}=u t^{\frac{2 \alpha}{5}} .
$$

Hence, from the symmetry $X_{2}$, we get the group-invariant solution

$$
u=t^{-\frac{2 \alpha}{5}} f(\xi), \quad \xi=x t^{-\frac{\alpha}{5}},
$$

in Eq. (15), $f$ is an arbitrary function of $\xi$. Using Eq. (15), Eq. (1) is transformed to a special nonlinear ODE of fractional order.

Consider the following theorem

Theorem 3.1. The similarity transformation Eq. (15) reduces Eq. (1) to the nonlinear ODE of fractional order as below:

$$
\left(P_{\frac{5}{\alpha}}^{1-\frac{7 \alpha}{5}, \alpha} f\right)(\xi)+\beta f^{2} f_{\xi}+\gamma f_{\xi \xi \xi \xi \xi}=0
$$

with the EK fractional differential operator [22]

$$
\left(P_{\beta}^{\xi^{2}, \alpha} f\right)=\Pi_{j=0}^{n-1}\left(\xi^{2}+j-\frac{1}{\beta} \frac{d}{d \xi}\right)\left(K_{\beta}^{\xi^{2}+\alpha, n-\alpha} f\right)(\xi),
$$

$$
n= \begin{cases}{[\alpha]+1,} & \alpha \neq \mathbb{N}, \\ \alpha, & \alpha \in \mathbb{N},\end{cases}
$$

where

$\left(K_{\beta}^{\xi^{2}, \alpha} f\right)(\xi)= \begin{cases}\frac{1}{\Gamma(\alpha)} \int_{1}^{\infty}(u-1)^{\alpha-1} u^{-\left(\xi^{2}+\alpha\right)} f\left(\xi u^{\frac{1}{\beta}}\right) d u, & \alpha>0, \\ f(\xi), & \alpha=0,\end{cases}$

is the EK fractional integral operator $[74,75]$.

Proof. Let $n-1<\alpha<1, n=1,2,3, \ldots$. Based on the RL fractional derivative in Eq. (15), we get

$$
\frac{\partial^{\alpha} u}{\partial t^{\alpha}}=\frac{\partial^{n}}{\partial t^{n}}\left[\frac{1}{\Gamma(n-\alpha)} \int_{1}^{t}(t-s)^{n-\alpha-1} s^{\frac{\alpha}{5}} f\left(x s^{-\left(\frac{\alpha}{5}\right)}\right) d s\right] .
$$

Let $v=\frac{t}{s}, d s=-\frac{t}{v^{2}} d v$. Thus, Eq. (20) becomes

$\frac{\partial^{\alpha} u}{\partial t^{\alpha}}=\frac{\partial^{n}}{\partial t^{n}}\left[t^{n-\frac{7 \alpha}{5}} \frac{1}{\Gamma(n-\alpha)} \int_{1}^{\infty}(v-1)^{n-\alpha-1} v^{-\left(n+1-\frac{7 \alpha}{5}\right)} f\left(\xi v^{\frac{\alpha}{5}}\right) d v\right]$.

Applying EK fractional integral operator Eq. (19) in Eq. (21), we get

$$
\frac{\partial^{\alpha} u}{\partial t^{\alpha}}=\frac{\partial^{n}}{\partial t^{n}}\left[t^{n-\frac{7 \alpha}{5}}\left(K_{\frac{5}{\alpha}}^{1-\frac{2 \alpha}{5}, n-\alpha} f\right)(\xi)\right] .
$$

We simplify the right hand side of Eq. (22). Consider $\xi=$ $x t^{-\frac{\alpha}{5}}, \phi \in(0, \infty)$, we acquire

$$
t \frac{\partial}{\partial t} \phi(\xi)=t x\left(-\frac{\alpha}{5}\right) t^{-\frac{\alpha}{5}-1} \phi^{\prime}(\xi)=-\frac{\alpha}{5} \xi \frac{\partial}{\partial \xi} \phi(\xi) .
$$

Hence,

$$
\begin{aligned}
& \frac{\partial^{n}}{\partial t^{n}}\left[t^{n-\frac{7 \alpha}{5}}\left(K_{\frac{5}{\alpha}}^{1-\frac{2 \alpha}{5}, n-\alpha} f\right)(\xi)\right]= \\
& \frac{\partial^{n-1}}{\partial t^{n-1}}\left[\frac{\partial}{\partial t}\left(t^{n-\frac{7 \alpha}{5}}\left(K_{\frac{5}{\alpha}}^{1-\frac{2 \alpha}{5}, n-\alpha} f\right)(\xi)\right)\right] \\
& = \\
& \frac{\partial^{n-1}}{\partial t^{n-1}}\left[t^{n-\frac{7 \alpha}{5}-1}\left(n-\frac{7 \alpha}{5}-\frac{\alpha}{5} \xi \frac{\partial}{\partial \xi}\right)\left(K_{\frac{5}{\alpha}}^{1-\frac{2 \alpha}{5}, n-\alpha} f\right)(\xi)\right] .
\end{aligned}
$$

Repeating $n-1$ times, we have

$$
\begin{aligned}
& \frac{\partial^{n}}{\partial t^{n}}\left[t^{n-\frac{7 \alpha}{5}}\left(K_{\frac{5}{\alpha}}^{1-\frac{2 \alpha}{5}, n-\alpha} f\right)(\xi)\right]= \\
& \frac{\partial^{n-1}}{\partial t^{n-1}}\left[\frac{\partial}{\partial t}\left(t^{n-\frac{7 \alpha}{5}}\left(K_{\frac{5}{\alpha}}^{1-\frac{2 \alpha}{5}, n-\alpha} f\right)(\xi)\right)\right]
\end{aligned}
$$




$$
\begin{aligned}
& \frac{\partial^{n-1}}{\partial t^{n-1}}\left[t^{n-\frac{7 \alpha}{5}-1}\left(n-\frac{7 \alpha}{5}-\frac{\alpha}{5} \xi \frac{\partial}{\partial \xi}\right)\right. \\
& \left.\times\left(K_{\frac{5}{\alpha}}^{1-\frac{2 \alpha}{5}, n-\alpha} f\right)(\xi)\right] . \\
& =t^{-\frac{7 \alpha}{5}} \Pi_{j=0}^{n-1}\left[\left(1-\frac{7 \alpha}{5}+j-\frac{\alpha}{5} \xi \frac{\partial}{\partial \xi}\right)\right. \\
& \left.\times\left(K_{\frac{5}{\alpha}}^{1-\frac{2 \alpha}{5}, n-\alpha} f\right)(\xi)\right] .
\end{aligned}
$$

Applying EK fractional differential operator Eq. (17) in Eq. (25), we get

$$
\begin{aligned}
& \frac{\partial^{n} u}{\partial t^{n}}\left[\left(t^{n-\frac{7 \alpha}{5}}\left(K_{\frac{5}{\alpha}}^{1+\frac{\alpha}{5}, n-\alpha} f\right)(\xi)\right)\right]= \\
& t^{-\frac{7 \alpha}{5}}\left(P_{\frac{5}{\alpha}}^{1-\frac{5 \alpha}{6}, \alpha} f\right)(\xi)
\end{aligned}
$$

Substituting Eq. (26) into Eq. (22), we get

$$
\frac{\partial^{\alpha} u}{\partial t^{\alpha}}=t^{-\frac{7 \alpha}{5}}\left(P_{\frac{5}{\alpha}}^{1-\frac{7 \alpha}{5}, \alpha} f\right)(\xi)
$$

Thus, Eq. (1) can be reduced into a fractional order ODE

$$
\left(P_{\frac{5}{\alpha}}^{1-\frac{7 \alpha}{5}, \alpha} f\right)(\xi)+\beta f^{2} f_{\xi}+\gamma f_{\xi \xi \xi \xi \xi}=0
$$

The proof of the theorem is completed.

\section{Conservation laws}

We now construct the Cls for Eq. (1). We start with some definitions. The RL left-sided time-fractional derivative given by

$$
o D_{t}^{\alpha} u=D_{t}^{n}\left(o I^{n-\alpha} u\right)
$$

where $D_{t}$ is the total differential operator with respect to $t, n=[\alpha]+1$, and $o I^{n-\alpha} u$ represents the left sided timefractional integral of $n-\alpha$ order given by

$$
\left(o I^{n-\alpha} u\right)(x, t)=\frac{1}{\Gamma(n-\alpha)} \int_{0}^{t} \frac{u(\theta, x)}{(t-\theta)^{1-n+\alpha}} d \theta .
$$

In Eq. (30), $\Gamma(z)$ represents Gamma function.
A Cls for Eq. (1) is represented as

$$
D_{t}\left(C^{t}\right)+D_{x}\left(C^{x}\right)=0
$$

where $C^{t}=C^{t}(x, t, u, \ldots), C^{x}=C^{x}(x, t, u, \ldots)$, and Eq. (31) holds for all solutions $u(x, t)$ of the Eq. (1).

We now apply Ibragimov method [48] for constructing the Cls of Eq. (1). Lagrangian for Eq. (1) can be presented as

$$
L=v(x, t)\left(\frac{\partial^{\alpha} u}{\partial t^{\alpha}}+\beta u^{2} u_{x}+\gamma u_{x x x x x}\right)
$$

where $v(x, t)$ is another dependent variable. The EulerLagrange operator $[45,46]$ is

$$
\begin{gathered}
\frac{\delta}{\delta u}=\frac{\partial}{\partial u}+\left(D_{t}^{\alpha}\right)^{\star} \frac{\partial}{\partial D_{t}^{\alpha} u}-D_{x} \frac{\partial}{\partial u_{x}}+D_{x x}^{2} \frac{\partial}{\partial u_{x x}}-D_{x x x}^{3} \frac{\partial}{\partial u_{x x x}} \\
+D_{x x x x}^{4} \frac{\partial}{\partial u_{x x x x}}-D_{x x x x x}^{5} \frac{\partial}{\partial u_{x x x x x}},
\end{gathered}
$$

where $\left(D_{t}^{\alpha}\right)^{\star}$ is the adjoint operator of $\left(D_{t}^{\alpha}\right)$. The adjoint equation to Eq. (1) is given by [48]

$$
\frac{\delta L}{\delta u}=0
$$

Consider two independent variables $x, t$ and one dependent variable $u(x, t)$, we have that

$$
\bar{X}+D_{t}\left(\xi^{2}\right) l+D_{x}\left(\xi^{1}\right) l=W \frac{\delta}{\delta u}+D_{t} N^{t}+D_{x} N^{x}
$$

in Eq. (35), $l$ represent the identity operator, $\frac{\delta}{\delta u}$ is the EulerLagrangian operator, $N^{t}$ and $N^{X}$ represent the Noether operation, $\bar{X}$ is defined by

$$
\begin{gathered}
\bar{X}=\xi^{2} \frac{\partial}{\partial t}+\xi^{1} \frac{\partial}{\partial x}+\eta \frac{\partial}{\partial u}+\eta_{\alpha}^{0} \frac{\partial}{\partial D_{t}^{\alpha} u}+\eta^{x} \frac{\partial}{\partial u_{x}}+\eta^{x x} \frac{\partial}{\partial u_{x x}} \\
+\eta^{x x x} \frac{\partial}{\partial u_{x x x}}+\eta^{x x x x} \frac{\partial}{\partial u_{x x x x}}+\eta^{x x x x x} \frac{\partial}{\partial u_{x x x x x}},
\end{gathered}
$$

and the Lie characteristic function $W$ is given by

$$
W=\eta-\xi^{2} u_{t}-\xi^{1} u_{x}
$$

When RL time-fractional derivative is used in Eq. (1), $N^{t}$ is defined by $[45,46]$

$$
N^{t}=\xi^{2} l+\sum_{k=0}^{n-1}(-1)^{k} o D^{\alpha-1-k}(W) D_{t}^{k} \frac{\partial}{\partial o D_{t}^{\alpha} u}-(-1)^{n}
$$




$$
\times J\left(W, D_{t}^{n} \frac{\partial}{\partial o D_{t}^{\alpha} u}\right) .
$$

With $J$ given by

$$
J(f, g)=\frac{1}{\Gamma(n-\alpha)} \int_{0}^{t} \int_{t}^{T} \frac{f\left(\xi^{2}, x\right) g(\mu, x)}{\left(\mu-\xi^{2}\right)^{\alpha+1-n}} d \mu d t .
$$

For Eq. (1), the operator $N^{x}$ is given by

$$
\begin{aligned}
N^{x}=\xi^{1} l & +W\left(\frac{\partial}{\partial u_{x}}-D_{x} \frac{\partial}{\partial u_{x x}}+D_{x}^{2} \frac{\partial}{\partial u_{x x x}}\right. \\
& \left.-D_{x}^{3} \frac{\partial}{\partial u_{x x x x}}+D_{x}^{4} \frac{\partial}{\partial u_{x x x x x}}\right)+D_{x}(W)\left(\frac{\partial}{\partial u_{x x}}\right. \\
& \left.-D_{x} \frac{\partial}{\partial u_{x x x}}+D_{x}^{2} \frac{\partial}{\partial u_{x x x x}}-D_{x}^{3} \frac{\partial}{\partial u_{x x x x x}}\right) \\
& +D_{x}^{2}(W)\left(\frac{\partial}{\partial u_{x x x}}-D_{x} \frac{\partial}{\partial u_{x x x x}}+D_{x}^{2} \frac{\partial}{\partial u_{x x x x x}}\right) \\
& +D_{x}^{3}(W)\left(\frac{\partial}{\partial u_{x x x x}}-D_{x} \frac{\partial}{\partial u_{x x x x x}}\right) \\
& +D_{x}^{4}\left(W_{i}\right) \frac{\partial}{\partial u_{x x x x x}}
\end{aligned}
$$

The invariance condition for any given generator $X$ of Eq. (1) and its solutions reads

$$
\left.\left(\bar{X} L+D_{t}\left(\xi^{2}\right) L+D_{x}\left(\xi^{1}\right) L\right)\right|_{E q .(1)}=0,
$$

and consequently the Cls of Eq. (1) can be written as

$$
D_{t}\left(N^{t} L\right)+D_{x}\left(N^{x} L\right)=0 .
$$

Now, we present the Cls for Eq. (1) using the basic definitions presented above. We consider two cases corresponding to the order of $\alpha$.

Case 1. When $\alpha \in(0,1)$, with the help of Eq. (38) and Eq. (39), the components of the conserved vectors are

$$
\begin{aligned}
C_{i}^{t}= & \xi^{2} L+(-1)^{0} o D_{t}^{\alpha-1}\left(W_{i}\right) D_{t}^{0} \frac{\partial L}{\partial\left(o D_{t}^{\alpha} u\right)}-(-1)^{1} \\
& \times J\left(W_{i}, D_{t}^{1} \frac{\partial L}{\partial\left(o D_{t}^{\alpha} u\right)}\right) \\
= & v_{o} D_{t}^{\alpha-1}\left(W_{i}\right)+J\left(W_{i}, v_{t}\right), \\
C_{i}^{x}=\xi^{1} l+ & W_{i}\left(\frac{\partial}{\partial u_{x}}-D_{x} \frac{\partial}{\partial u_{x x}}+D_{x}^{2} \frac{\partial}{\partial u_{x x x}}-D_{x}^{3} \frac{\partial}{\partial u_{x x x x}}\right. \\
+ & \left.D_{x}^{4} \frac{\partial}{\partial u_{x x x x x}}\right)+D_{x}\left(W_{i}\right)\left(\frac{\partial}{\partial u_{x x}}-D_{x} \frac{\partial}{\partial u_{x x x}}\right.
\end{aligned}
$$

$$
\begin{aligned}
& \left.+D_{x}^{2} \frac{\partial}{\partial u_{x x x x}}-D_{x}^{3} \frac{\partial}{\partial u_{x x x x x}}\right)+D_{x}^{2}\left(W_{i}\right)\left(\frac{\partial}{\partial u_{x x x}}\right. \\
& \left.-D_{x} \frac{\partial}{\partial u_{x x x x}}+D_{x}^{2} \frac{\partial}{\partial u_{x x x x x}}\right)+D_{x}^{3}\left(W_{i}\right)\left(\frac{\partial}{\partial u_{x x x x}}\right. \\
& \left.-D_{x} \frac{\partial}{\partial u_{x x x x x}}\right)+D_{x}^{4}\left(W_{i}\right) \frac{\partial}{\partial u_{x x x x x}}
\end{aligned}
$$

$$
\begin{aligned}
& =W_{i}\left(\beta u^{2} v+D_{x}^{4} \gamma v\right)+D_{x}\left(W_{i}\right)\left(-D_{x}^{3} \gamma v\right) \\
& \quad+D_{x}^{2}\left(W_{i}\right)\left(D_{x}^{2} \gamma v\right)-\gamma v_{x} D_{x}^{3}\left(W_{i}\right)+\gamma v D_{x}^{4}\left(W_{i}\right),
\end{aligned}
$$

where $i=1,2$ and the functions $W_{i}$ are given by

$$
W_{1}=-u_{x}, \quad W_{2}=-2 u \alpha-5 t u_{t}-\alpha x u_{x} .
$$

Case 2. When $\alpha \in(1,2)$, with the help of Eq. (38) and Eq. (39), the components of the conserved vectors are

$$
\begin{gathered}
C_{i}^{t}=\xi^{2} L+(-1)^{0} o D_{t}^{\alpha-1}\left(W_{i}\right) D_{t}^{0} \frac{\partial L}{\partial\left(o D_{t}^{\alpha} u\right)}-(-1)^{1} \\
J\left(W_{i}, D_{t}^{1} \frac{\partial L}{\partial\left(o D_{t}^{\alpha} u\right)}\right)+(-1)^{1} o D_{t}^{\alpha-2}\left(W_{i}\right) D_{t}^{1} \\
\frac{\partial L}{\partial\left(o D_{t}^{\alpha} u\right)}-(-1)^{1} J\left(W_{i}, D_{t}^{1} \frac{\partial L}{\partial\left(o D_{t}^{\alpha} u\right)}\right) \\
=v_{o} D_{t}^{\alpha-1}\left(W_{i}\right)+J\left(W_{i}, v_{t}\right)-v_{t} o D_{t}^{\alpha-2}\left(W_{i}\right)-J\left(W_{i}, v_{t t}\right)
\end{gathered}
$$

$$
\begin{aligned}
C_{i}^{x}=\xi^{1} l & +W_{i}\left(\frac{\partial}{\partial u_{x}}-D_{x} \frac{\partial}{\partial u_{x x}}+D_{x}^{2} \frac{\partial}{\partial u_{x x x}}-D_{x}^{3} \frac{\partial}{\partial u_{x x x x}}\right. \\
& \left.+D_{x}^{4} \frac{\partial}{\partial u_{x x x x x}}\right)+D_{x}\left(W_{i}\right)\left(\frac{\partial}{\partial u_{x x}}-D_{x} \frac{\partial}{\partial u_{x x x}}\right. \\
& \left.+D_{x}^{2} \frac{\partial}{\partial u_{x x x x}}-D_{x}^{3} \frac{\partial}{\partial u_{x x x x x}}\right)+D_{x}^{2}\left(W_{i}\right)\left(\frac{\partial}{\partial u_{x x x}}\right. \\
& \left.-D_{x} \frac{\partial}{\partial u_{x x x x}}+D_{x}^{2} \frac{\partial}{\partial u_{x x x x x}}\right)+D_{x}^{3}\left(W_{i}\right)\left(\frac{\partial}{\partial u_{x x x x}}\right. \\
& \left.-D_{x} \frac{\partial}{\partial u_{x x x x x}}\right)+D_{x}^{4}\left(W_{i}\right) \frac{\partial}{\partial u_{x x x x x}} \\
=W_{i} & \left(\beta u^{2} v+D_{x}^{4} \gamma v\right)+D_{x}\left(W_{i}\right)\left(-D_{x}^{3} \gamma v\right) \\
& +D_{x}^{2}\left(W_{i}\right)\left(D_{x}^{2} \gamma v\right)-\gamma v_{x} D_{x}^{3}\left(W_{i}\right)+\gamma v D_{x}^{4}\left(W_{i}\right),
\end{aligned}
$$

where $i=1,2$ and the functions $W_{i}$ are given by

$$
W_{1}=-u_{x}, \quad W_{2}=-2 u \alpha-5 t u_{t}-\alpha x u_{x} .
$$




\section{Explicit power series solutions}

Here, we investigate the exact analytic solutions via power series method [76] and symbolic computations [77] for Eq. (28). Set

$$
f(\xi)=\sum_{n=0}^{\infty} a_{n} \xi^{n}
$$

from Eq. (43), we can have

$$
\begin{aligned}
& f^{\prime}=\sum_{n=0}^{\infty} n a_{n} \xi^{n-1} \\
& f^{(5)}=\sum_{n=0}^{\infty} n(n-1)(n-2)(n-3)(n-4) a_{n} \xi^{n-5}
\end{aligned}
$$

Substituting Eqs. (44) into Eq. (28), we obtain

$$
\begin{aligned}
& \sum_{n=0}^{\infty} \frac{\Gamma\left(2-\frac{12 \alpha}{5}+\frac{n \alpha}{5}\right)}{\Gamma\left(2-\frac{7 \alpha}{5}+\frac{n \alpha}{5}\right.} a_{n} \xi^{n}+\beta \sum_{n=0}^{\infty} \sum_{n=0}^{\infty} a_{n} \xi^{n} \\
& \times \sum_{n=0}^{\infty}(n+1) a_{n+1} \xi^{n}+\gamma \sum_{n=0}^{\infty}(n+5)(n+4)(n+3) \\
& \times(n+2)(n+1) a_{n+5} \xi^{n}=0 .
\end{aligned}
$$

Comparing coefficients in Eq. (45) when $n=0$, we obtain

$$
a_{5}=\frac{1}{120 \gamma}\left(\frac{\Gamma\left(2-\frac{12 \alpha}{5}\right)}{\Gamma\left(2-\frac{7 \alpha}{5}\right)} a_{0}+\beta a_{0}^{2} a_{1}\right),
$$

when $n \geq 1$, we have

$$
\begin{aligned}
& a_{n+5}=\frac{1}{(n+5)(n+4)(n+3)(n+2)(n+1) \gamma} \\
& \times\left\{\frac{\Gamma\left(2-\frac{12 \alpha}{5}+\frac{n \alpha}{5}\right)}{\Gamma\left(2-\frac{7 \alpha}{5}+\frac{n \alpha}{5}\right.} a_{n}\right. \\
& \left.+\beta \sum_{k=0}^{n} \sum_{j=0}^{k} a_{j}(n-k+1) a_{n-k+1}\right\} .
\end{aligned}
$$

Thus, the power series solution for Eq. (28) can be represented in the form:

$$
\begin{aligned}
& f(\xi)=a_{0}+a_{1} \xi+a_{2} \xi^{2}+a_{3} \xi^{3}+a_{4} \xi^{4}+a_{5} \xi^{5} \\
& +\sum_{n=1}^{\infty} a_{n+5} \xi^{n+5} \\
& =a_{0}+a_{1} \xi+a_{2} \xi^{2}+a_{3} \xi^{3}+a_{4} \xi^{4}+\frac{1}{120 \gamma}\left(\frac{\Gamma\left(2-\frac{12 \alpha}{5}\right)}{\Gamma\left(2-\frac{7 \alpha}{5}\right)} a_{0}\right. \\
& \left.+\beta a_{0}^{2} a_{1}\right) \xi^{5}+\sum_{n=1}^{\infty} \frac{1}{(n+5)(n+4)(n+3)(n+2)(n+1) \gamma}
\end{aligned}
$$

$$
\begin{aligned}
& \times\left\{\frac{\Gamma\left(2-\frac{12 \alpha}{5}+\frac{n \alpha}{5}\right)}{\Gamma\left(2-\frac{7 \alpha}{5}+\frac{n \alpha)}{5}\right.} a_{n}+\beta \sum_{k=0}^{n} \sum_{j=0}^{k} a_{j}\right. \\
& \left.\times(n-k+1) a_{n-k+1}\right\} \xi^{n+5}
\end{aligned}
$$

Consequently, we acquire the exact power series solution for Eq. (28) as

$$
\begin{aligned}
& u(x, t)=a_{0} t^{-\frac{2 \alpha}{5}}+a_{1} x t^{-\frac{3 \alpha}{5}}+a_{2} x^{2} t^{-\frac{4 \alpha}{5}}+a_{3} x^{3} t^{-\alpha} \\
& +a_{4} x^{4} t^{-\frac{6 \alpha}{5}}+\frac{1}{120 \gamma}\left(\frac{\Gamma\left(2-\frac{12 \alpha}{5}\right)}{\Gamma\left(2-\frac{7 \alpha}{5}\right)} a_{0}+\beta a_{0}^{2} a_{1}\right) x^{5} t^{-\frac{7 \alpha}{5}} \\
& +\sum_{n=1}^{\infty} \frac{1}{(n+5)(n+4)(n+3)(n+2)(n+1) \gamma} \\
& \times\left\{\frac{\Gamma\left(2-\frac{12 \alpha}{5}+\frac{n \alpha}{5}\right)}{\Gamma\left(2-\frac{7 \alpha}{5}+\frac{n \alpha)}{5}\right.} a_{n}+\beta \sum_{k=0}^{n} \sum_{j=0}^{k} a_{j}\right. \\
& \left.\times(n-k+1) a_{n-k+1}\right\} x^{n+5} t^{-\frac{(n+7 \alpha)}{5}}
\end{aligned}
$$

\section{Physical interpretation of the power series solution for Eqs.}

\section{(47)}

In order to have clear and proper understanding of the physical properties of the power series solution, the 3-D, 2-D and contour plots for the solution Eqs. (47), are plotted in Figures 1-4 by using suitable parameter values.

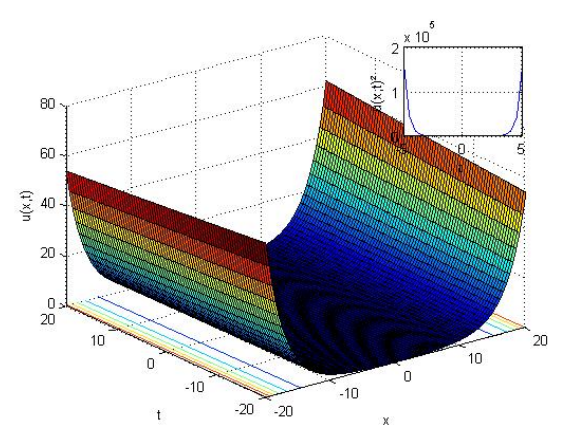

Figure 1: 3D plot of (47) $a_{0}=a_{1}=a_{2}=1, a_{3}=0.5, a_{4}=1.7, \beta=$ 2, $\gamma=1, \alpha=0.5, \Gamma=0.85$ 


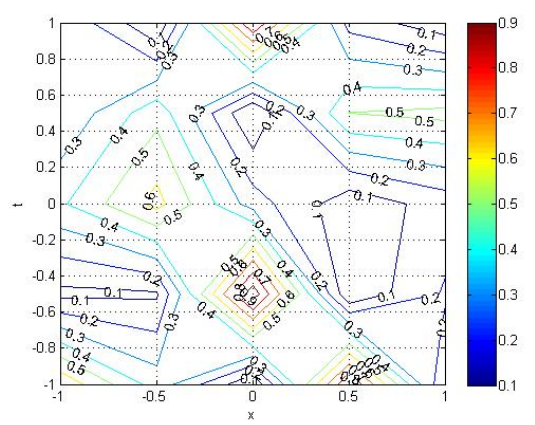

Figure 2: Contour plot of (47) $a_{0}=a_{1}=a_{2}=1, a_{3}=0.5, a_{4}=$ 1.7, $\beta=2, \gamma=1, \alpha=0.5, \Gamma=0.85$

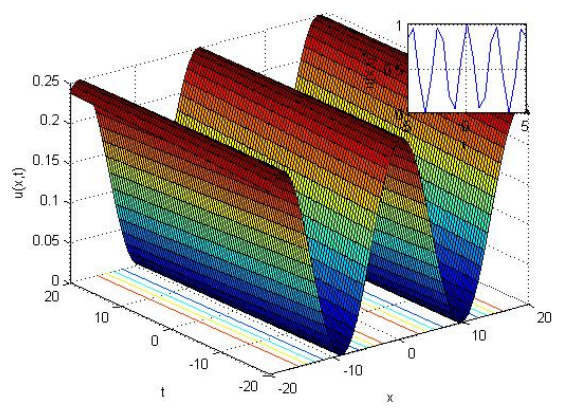

Figure 3: 3D plot of (47) $a_{0}=a_{1}=a_{2}=0.8, a_{3}=a_{4}=1, \beta=$ $1.2, \gamma=3, \alpha=0.9, \Gamma=0.1$

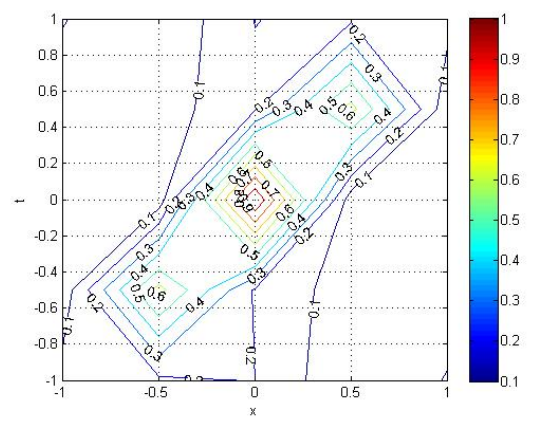

Figure 4: Contour plot of (47) $a_{0}=a_{1}=a_{2}=0.8, a_{3}=a_{4}=1, \beta=$ $1.2, \gamma=3, \alpha=0.9, \Gamma=0.1$

\section{Concluding remarks}

In this research, we analyzed time fractional SMK by means of Lie symmetry analysis using the RL derivative. We reduced the governing equation to a nonlinear ODE of fractional order. The obtained fractional ODE was solved using a power series technique. Ibragimov's nonlocal conservation theorem was applied to establish Cls for the governing equation. Some 3-D, 2-D, and contour plots were also presented.

\section{References}

[1] Olver P.J., Application of Lie Group to Differential Equation, 1986, Springer, New York

[2] Ovsiannikov L.V., Group Analysis of Differential Equations, 1982, Academic Press, New York

[3] Lie S., On integration of a class of linear partial differential equations by means of definite integrals, 1881, Arch. Math. Log., 6(3), 328-368.

[4] Bluman G.W., Kumei S., Symmetries and Differential Equations, 1989, Springer, New York

[5] Ibragimov N.H., Handbook of Lie Group Analysis of Differential Equations, 1994, vol. 1, CRC Press, Boca Raton

[6] Wang X.B., Tian S.F., Qin C.Y., Zhang T.T., Lie symmetry analysis, conservation laws and exact solutions of the generalized time fractional Burgers equation, Europhys. Lett., 2016, 114(2), 20003

[7] Wang X.B., Tian S.F., Qin C.Y., Zhang T.T., Lie symmetry analysis, conservation laws and analytical solutions of a time-fractional generalized KdV-type equation. J. Nonlinear Math. Phys., 2017, 24, 516-530.

[8] Feng L.L., Tian S.F., Wang X.B., Zhang T.T., Lie Symmetry Analysis, Conservation Laws and Exact Power Series Solutions for Time-Fractional Fordy-Gibbons Equation. Commun. Theor. Phys., 2016, 66(3), 321

[9] Tu J.M., Tian S.F, Xu M.J., Zhang T.T., On Lie symmetries, optimal systems and explicit solutions to the Kudryashov-Sinelshchikov equation, Appl. Math. Comput., 2016, 275, 345-352.

[10] Ma P.L., Tian S.F., Zhang T.T., On symmetry-preserving difference scheme to a generalized Benjamin equation and third- order Burgers equation, Appl. Math. Lett., 2015, 50, 146-152.

[11] Inc M., Yusuf A., Aliyu A.I., Baleanu D., Lie symmetry analysis and explicit solutions for the time fractional generalized Burgers-Huxley equation, Opt. Quant. Electron., 2018, 50, 94

[12] Wang G.W., Xu T.Z., Invariant analysis and explicit solutions of the time fractional nonlinear perturbed Burgers equation, Nonlinear Anal., 2015, 20, 570-584.

[13] Wang G.W., Kara A.H., Fakhar K., Symmetry analysis and conservation laws for the class of time-fractional nonlinear dispersive equation, Nonlinear Dyn., 2015, 82,281-287.

[14] Baleanu D., Inc M., Yusuf A., Aliyu A.I., Time Fractional Third- Order Evolution Equation: Symmetry Analysis, Explicit Solutions, and Conservation Laws, J. Comput. Nonlinear Dynam., 2017, 13(2), 021011

[15] Baleanu D., Inc M., Yusuf A., Aliyu A.I., Lie symmetry analysis, exact solutions and conservation laws for the time fractional Caudrey-Dodd-Gibbon-Sawada-Kotera equation, Commun Nonlinear Sci. Numer. Simulat., 2018, 59, 222-234.

[16] Jefferson G.F., On the second-order approximate symmetry classification and optimal systems of subalgebras for a forced Korteweg-de Vries equation, Commun. Nonlinear Sci. Numer. Simul., 2013, 18, 2340-2358.

[17] Tchier F., Aliyu A.I., Yusuf A., Inc M., Dynamics of solitons to the ill-posed Boussinesq equation, Eur. Phys. J. Plus., 2017, 132136.

[18] Diethelm K., The Analysis of Fractional Differential Equations, 2010, Springer, Berlin

[19] Miller K.S., Ross B., An Introduction to the Fractional Calculus and Fractional Differential Equations, 1993, Wiley, New York 
[20] Podlubny I., Fractional Differential Equations, 1999, Academic Press, San Diego

[21] Oldham K.B., Spanier J., The Fractional Calculus, 1974, Academic Press, San Diego

[22] Kiryakova V., Generalised Fractional Calculus and Applications, Pitman Res. Notes in Math., 1994, 301.

[23] El-Sayed A.M.A.,Gaber M., The Adomian decomposition method for solving partial differential equations of fractal order in finite domains, Phys. Lett. A., 2006, 359, 175-182.

[24] Chen Y., An H.L., Numerical solutions of coupled Burgers equations with time and space fractional derivatives, Appl. Math. Comput., 2008, 200, 87-95.

[25] Inc M., Yusuf A., Aliyu A.I., Baleanu D., Soliton structures to some time-fractional nonlinear differential equations with conformable derivative, Opt, Quant, Electron., 2018, 50, 20

[26] Odibat Z., Momani S., A generalized differential transform method for linear partial differential equations of fractional order, Appl. Math. Lett., 2008, 21, 194-199.

[27] Li X., Chen W., Analytical study on the fractional anomalous diffusion in a half-plane, J. Phys. A, Math. Theor., 2010, 43(49), 495206

[28] He T.H., A coupling method of a homotopy technique and a perturbation technique for non-linear problems, Int. J. Non-Linear Mech., 2000, 35, 37-43.

[29] Inc M., Yusuf A., Aliyu A.I., Baleanu D., Lie symmetry analysis, explicit solutions and conservation laws for the space-time fractional nonlinear evolution equations, Physica A., 2018, 496, 371-383.

[30] Zhang S., Zhang H.Q., Fractional sub-equation method and its applications to nonlinear fractional PDEs, Phys. Lett. A., 2011, 375, 1069-1073.

[31] Guo S., Mei L.Q., Li Y., Sun Y.F., The improved fractional subequation method and its applications to the space-time fractional differential equations in fluid mechanics, Phys. Lett. A., 2012, 376, 407-411.

[32] Lu B., Bäcklund transformation of fractional Riccati equation and its applications to nonlinear fractional partial differential equations. Phys. Lett. A., 2012, 376, 2045-2048.

[33] Jumarie G., Modiffed Riemann-Liouville derivative and fractional Taylor series of non differentiable functions further results, Comput. Math. Appl., 2006, 51, 1367-1376.

[34] Jumarie G., Cauchy's integral formula via the modified RiemannLiouville derivative for analytic functions of fractional order Appl. Math. Lett.,2010, 23, 1444-1450.

[35] Sahadevan R., Bakkyaraj T., Invariant analysis of time fractional generalized Burgers and Korteweg-de Vries equations, J. Math. Anal. Appl., 2012, 393, 341-347.

[36] Wang G.W., Liu X.Q., Zhang Y.Y., Lie symmetry analysis to the time fractional generalized fffth-order KdV equation, Commun. Nonlinear Sci. Numer. Simul., 2013, 18, 2321-2326.

[37] Inc M., Yusuf A. , Aliyu A.I. , Baleanu D., Time-fractional CahnAllen and time-fractional Klein-Gordon equations: Lie symmetry analysis, explicit solutions and convergence analysis, Physica A., 2018, 493, 94-106.

[38] Baleanu D., Inc M. , Yusuf A., Aliyu A.I., Lie symmetry analysis, exact solutions and conservation laws for the time fractional modiffied Zakharov-Kuznetsov equation, Nonlinear Anal.: Model. and Contr., 2017 , 22(6), 861-876.

[39] Buckwar E., Luchko Y. Invariance of a partial differential equation of fractional order under the Lie group of scaling transfor- mations, J. Math. Anal. Appl., 1998, 227 81-97.

[40] Djordjevic V.D., Atanackovic T.M., Similarity solutions to nonlinear heat conduction and Burgers/Korteweg-de Vries fractional equations, J. Comput. Appl. Math. 212, 2008, 701-714.

[41] Liu H.Z., Complete group classifications and symmetry reductions of the fractional fifth-order KdV types of equations, Stud. Appl. Math., 2013, 131, 317-330.

[42] Olver P.J., Application of Lie Groups to Differential Equations, 1993, New York, Springer-Verlag

[43] Ibragimov N.H., Elementary Lie Group Analysis and Ordinary Differential Equations, 1999, John Wiley \& Sons, Chichester

[44] Baleanu D., Inc M., Yusuf A., Aliyu A.I., Space-time fractional Rosenou-Haynam equation: Lie symmetry analysis, explicit solutions and conservation laws, Adv. Diff. Eq., 2018, 2018, 46

[45] Noether E., Invariante Variations Probleme, Transp. Theor. Stat. Phys.,1971, 1(3), 186-207.

[46] Kara A.H., Mahomed F.M., Noether-type symmetries and conservation laws via partial Lagragians, Nonlinear Dyn., 2006, 45, 367-383.

[47] Anco S.C., Bluman G.W., Direct construction method for conservation laws of partial differential equations. Part I: examples of conservation law classifications, Eur. J. Appl. Math., 2002, 13, 545-566.

[48] Ibragimov N.H., A new conservation theorem, J. Math. Anal. Appl., 2007, 28, 333-311.

[49] Wang G.W., Xu T.Z., Group Analysis and New Explicit Solutions of Simplified Modified Kawahara Equation with Variable Coefficients, Abstr. Appl. Anal., 2013, 2013, 139160

[50] Igor L., Julio C.F., Sampaio S. Conservation laws for Kawahara equations, Matematica Aplicada E Computacional, 2012, 17(21), 287-292.

[51] Hounkonnou M.N., Mahaman M.K., Symmetry, integrability and solutions of the Kawahara equation, SUT J. Math., 2008, 44(1), 39-53.

[52] Wang X.B., Shou-Fu T., Mei-Juan X., Tian-Tian Z. On integrability and quasi-periodic wave solutions to a (3+1)-dimensional generalized KdV-like model equation, Appl. Math. Comput., 2016, 283, 216-233.

[53] Tu J.M., Tian S.F., XU M.J., Ma P.L., Zhang T.T., On periodic wave Solutions with asymptotic behaviors to a $(3+1)$-dimensional generalized B-type Kadomtsev-Petviashvili equation in fluid dynamics, Comput. \& Math. Appl., 2016, 72, 2486-2504.

[54] Xu M.J., Tian S.F., Tu J.M., Zhang T.T., Bäcklund transformation, inffnite conservation laws and periodic wave solutions to a generalized (2+1)-dimensional Boussinesq equation, Nonlinear Anal.: Real World Applications., 2016, 31, 388-408.

[55] Wangs X.B., Tian S.F., Qin C.Y., Zhang T.T., Zhang. Characteristics of the solitary waves and rogue waves with interaction phenomena in a generalized (3+1)-dimensional Kadomtsev- Petviashvili equation, Appl. Math. Lett., 2017, 72, 58-64.

[56] Tu J.M., Tian S.F, Xu M.J.,Zhang T.T., Quasi-periodic Waves and SolitaryWaves to a Generalized KdV-Caudrey-Dodd-Gibbon Equation from Fluid Dynamics, Taiwanese J. Math., 2016, 20, 823-848.

[57] Wang X.B., Tian S.F., Qin C.Y., Zhang T.T. Dynamics of the breathers, rogue waves and solitary waves in the $(2+1)$ - dimensional Ito equation, Appl. Math. Lett., 2017, 68, 40-47.

[58] Tian S.F., Initial-boundary value problems for the general coupled nonlinear Schrödinger equation on the interval via the Fokas method, J. Diff. Eq., 2017, 262(1), 506-558. 
[59] Bridges T.J., Multi-symplectic structures and wave propagation, Math. Proc. Cambr. Phil. Soc., 1997, 121,147-190.

[60] Hu W.P., Deng Z.C., Zhang Y., Multi-symplectic method for peakon-antipeakon collision of quasi-Degasperis-Procesi equation, Comp. Phys. Comm., 2014,185, 2020-2028.

[61] Hu W.P., Deng Z.C., Han S.M., Zhang W.R., Generalized Multisymplectic Integrators for a Class of Hamiltonian Nonlinear Wave PDEs, J. Comp. Phys., 2013, 235, 394-406.

[62] Hu W.P., Deng Z.C., Yin T.T., Almost structure-preserving analysis for weakly linear damping nonlinear Schrodinger equation with periodic perturbation, Comm. Nonlinear Sci. Numer. Simul., 2017, 42, 298-312.

[63] HuW., Deng Z., Competition between geometric dispersion and viscous dissipation in wave propagation of KdV-Burgers equation, J. Vibr. Contr., 2015, 21, 2937-2945.

[64] Hu W., Song M., Deng Z., Yin T., Wei B., Axial dynamic buckling analysis of embedded single-walled carbon nanotube by complex structure-preserving method, Applied Mathematical Modelling., 2017, 52, 15-27.

[65] Hu W.P., Deng Z.C., Chaos in embedded fluid-conveying single walled carbon nanotube under transverse harmonic load series, Nonlinear Dyn., 2015, 79, 325-333.

[66] Hu W.P., Deng Z.C., Wang B., Ouyang H.J., Chaos in an embedded single-walled carbon nanotube, Nonlinear Dyn., 2013, 72, 389-398.

[67] HuW., Song M., Deng Z., Zou H., Wei B., Chaotic region of elastically restrained single-walled carbon nanotube, Chaos, Solit. Fract., 2017, 27, 023118.
[68] Hu W., Song M., Deng Z., Energy dissipation/transfer and stable attitude of spatial on-orbit tethered system, J. Sound Vibr., 2018, 412, 58-73.

[69] Hu W., Li Q., Jiang X., Deng Z., Coupling dynamic behaviors of spatial flexible beam with weak damping, Int. J. Numer. Meth. Eng., 2017, 111, 660-675.

[70] Kiryakova V., Generalised Fractional Calculus and Applications, Pitman Res. Notes Math., 1994, 301, Longman, London

[71] Podlubny I., Fractional Differential Equations, 1999, San Diego, Academic Press

[72] Gazizov R.K., A.A. Kasatkin., S.Y. Lukashcuk., Symmetry properties of fractional diffusion equations, Phys. Scr., 2009, 136, 014-016.

[73] Gazizov R.K., Kasatkin A.A., Lukashcuk S.Y., Continuous transformation groups of fractional differential equations, Vestnik, USATU., 2007, 9, 125-135.

[74] Sahadevan R., Bakkyaraj T., Invariant analysis of time fractional generalized Burgers and Korteweg-de Vries equations, J. Math. Anal. Appl., 2012, 393, 341-347.

[75] Wang G.W., Xu T.Z., Invariant analysis and exact solutions of nonlinear time fractional Sharma-Tasso-Olver equation by Lie group analysis, Nonlinear Dyn., 2014, 76, 571-580.

[76] Galaktionov V.A., Svirshchevskii S.R., Exact Solutions and Invariant Subspaces of Nonlinear Partial Differential Equations in Mechanics and Physics, 2006, Chapman and Hall/CRC, Boca Raton, Florida

[77] Tian S.F., Zhang H.Q., On the integrability of a generalized variable-coeffcient Kadomtsev-Petviashvili equation, J. Phys. A: Math. Theor., 2012, 45, 055203. 